\title{
On Gradient Solitons of the Ricci-Harmonic Flow
}

\author{
Hong Xin GUO \\ School of Mathematics and Information Science, Wenzhou University, \\ Wenzhou 325035, P. R. China \\ E-mail:guo@wzu.edu.cn
}

\begin{abstract}
Robert PHILIPOWSKI Anton THALMAIER
Mathematics Research Unit, University of Luxembourg, 6 rue Richard Coudenhove-Kalergi, L-1359 Luxembourg, Grand-Duchy of Luxembourg

E-mail:robert.philipowski@uni.luａnton.thalmaier@uni.lu
\end{abstract}

\begin{abstract}
In this paper, we study gradient solitons to the Ricci flow coupled with harmonic map heat flow. We derive new identities on solitons similar to those on gradient solitons of the Ricci flow. When the soliton is compact, we get a classification result. We also discuss the relation with quasi-Einstein manifolds.
\end{abstract}

Keywords Ricci flow, harmonic map heat flow, gradient solitons, quasi-Einstein manifold

MR(2010) Subject Classification 53C44, 53C43, 58E20

\section{Introduction}

In recent years, various geometric flows have been studied extensively. In [11], Müller introduced a new flow generalizing Hamilton's Ricci flow, called the Ricci flow coupled with harmonic map heat flow, which we call Ricci-Harmonic flow or RH flow for short in the sequel. In addition to sharing many good properties with the Ricci flow, the RH flow is less singular than the Ricci flow or the harmonic map flow alone. See [11] for details, as well as [8, 13] for results and examples of this coupled flow. In [6], we developed a stochastic approach to the harmonic map heat flow on manifolds with time-dependent metric; there, however, the evolution of the metric is not coupled to the heat flow.

We start by briefly introducing the setting and our notations. Let $(M, g(t))$ and $(N, h)$ be two Riemannian manifolds of dimension $m$ and $n$, respectively. The metric $g(t)$ evolves along the $\mathrm{RH}$ flow equation on $M$, and $h$ is a static metric on the target manifold $N$. In addition, let $c(t)$ denote a nonnegative time-dependent constant, $\phi(t): M \rightarrow N$ a time-dependent map and $\tau(\phi)=\operatorname{trace} \nabla d \phi$ the tension field of $\phi$.

Received July 25, 2014, accepted March 31, 2015

The first author is supported by NSFC (Grant No. 11171143) and Zhejiang Provincial Natural Science Foundation of China (Project No. LY13A010009 and LY14A010021); the second and the third author are supported by the Fonds National de la Recherche Luxembourg (OPEN Project GEOMREV) 
The $\mathrm{RH}$ flow is the coupled system

$$
\left\{\begin{array}{l}
\frac{\partial g}{\partial t}=-2 \mathrm{Rc}+2 c(t) d \phi \otimes d \phi \\
\frac{\partial \phi}{\partial t}=\tau(\phi)
\end{array}\right.
$$

where $d \phi \otimes d \phi=\phi^{*} h$ is the pullback of $h$ via $\phi$. In this paper, we use both notations to respect the original reference.

As in Hamilton's Ricci flow, it is important to study self-similar solutions, i.e., solutions that change only by diffeomorphisms and rescaling. The simplest self-similar solutions are the gradient solitons which associate canonical solutions to the RH flow, see [11]. There are two methods to study gradient solitons: one is to treat them as static manifolds, and the other is to associate them with canonical solutions to the flow.

In this article, we will study solitons with respect to a fixed metric on $M$. The following definition is taken from [11].

Definition 1.1 Assume that $(M, g)$ and $(N, h)$ are two static Riemannian manifolds and let $\phi: M \rightarrow N$ be a smooth map. If there is a nonnegative constant $c$, a smooth function $f: M \rightarrow \mathbb{R}$ and a constant $\sigma$ such that

$$
\begin{aligned}
& \mathrm{Rc}-c d \phi \otimes d \phi+\operatorname{Hess} f+\sigma g=0, \\
& \tau(\phi)-d \phi(\nabla f)=0,
\end{aligned}
$$

then $((M, g),(N, h), \phi, f, \sigma)$ is called a gradient $\mathrm{RH}$ soliton and $f$ a potential function. The soliton is called shrinking, steady or expanding for $\sigma<0, \sigma=0$ or $\sigma>0$, respectively.

As in Ricci flow, on a gradient $\mathrm{RH}$ soliton $((M, g),(N, h), \phi, f, \sigma)$ one can construct a selfsimilar solution to (1.1) which changes only by diffeomorphisms and rescalings. Here the diffeomorphism is generated by $\nabla f$ and the rescaling factor is determined by $\sigma$.

We now list examples of gradient $\mathrm{RH}$ solitons.

(i) Gradient RH solitons cover the case of gradient solitons to the Ricci flow. Indeed, letting $\phi$ be a constant map in Definition 1.1, the RH soliton equations reduce to the gradient Ricci soliton equation.

(ii) Let $\phi: M \rightarrow N$ be a harmonic map and $f=$ const. Then the $\mathrm{RH}$ soliton equations reduce to the condition

$$
\mathrm{Rc}-c d \phi \otimes d \phi+\sigma g=0,
$$

which generalizes the notion of Einstein manifold. For instance, let $(M, g)$ be an Einstein manifold, i.e., $\mathrm{Rc}=\alpha g$ for some constant $\alpha$, and Id: $M \rightarrow M$ the identity map which is trivially harmonic. Then $((M, g),(M, g)$, Id, const, $\sigma)$ satisfies (1.4) with $\sigma=c-\alpha$. Likewise, one may take $(N, h)=(\mathbb{R}$, can $)$ with $\phi$ and $f$ constant functions, then the RH soliton equations reduce to $\mathrm{Rc}+\sigma g=0$.

(iii) Assume $(M, g)$ is a Riemannian manifold with strictly positive Ricci curvature. Then it is known that $\mathrm{Id}:(M, g) \rightarrow(M, \mathrm{Rc})$ is harmonic, see [1, Chapter 10, Section 2]. Hence, (1.4) holds for $c=1$ and $\sigma=0$. Thus $((M, g),(M, \mathrm{Rc})$, Id, const, 0$)$ is a steady $\mathrm{RH}$ soliton with $c=1$.

Recently, there have been some papers generalizing gradient Ricci solitons, for instance $[2,12]$ on almost Ricci solitons, [3] on quasi-Einstein manifolds and [7] on generalized quasi- 
Einstein manifolds. The gradient RH soliton is a generalization of gradient Ricci solitons in a different way.

In this article, we will use Ricci flow techniques to study gradient RH solitons. We note that in $[4,14]$ some results on $\mathrm{RH}$ solitons were obtained.

\section{Identities on Gradient RH Solitons}

In this section, we derive first identities on gradient $\mathrm{RH}$ solitons. To simplify notations, we define a symmetric two tensor

$$
\mathscr{S} \doteqdot \mathrm{Rc}-c d \phi \otimes d \phi
$$

and $S \doteqdot \operatorname{trace} \mathscr{S}=R-c|d \phi|^{2}$ where $|d \phi|^{2}=g^{i j} h_{\alpha \beta} \partial_{i} \phi^{\alpha} \partial_{j} \phi^{\beta}$.

We first derive a formula generalizing the corresponding formula in gradient Ricci solitons.

Lemma 2.1 On a gradient $\mathrm{RH}$ soliton, we have

$$
\mathscr{S}(\nabla f, \cdot)=\frac{1}{2} d S
$$

Proof Identity (2.1) can be found implicitly in [10, Chapter 4]. For the sake of completeness, we include a different proof here. The proof is a direct calculation based on the soliton equations (1.2) and (1.3). Tracing (1.2) with respect to the metric $g$, we have

$$
R-c|d \phi|^{2}+\Delta f+m \sigma=0 .
$$

Taking divergence of (1.2), we get

$$
\operatorname{Div}(\operatorname{Rc})-c \operatorname{Div}(d \phi \otimes d \phi)+\operatorname{Div}(\operatorname{Hess} f)=0 .
$$

In the above, by the contracted second Bianchi identity, we have $\operatorname{Div}(\mathrm{Rc})=d R / 2$. For the second term, we have

$$
\operatorname{Div}(d \phi \otimes d \phi)=\tau(\phi) d \phi+\frac{1}{2} d|d \phi|^{2} .
$$

Identity (2.4) will be proved in the appendix using local coordinates. By Bochner's formula and $(2.2)$, the third term in $(2.2)$ writes as

$$
\operatorname{Div}(\operatorname{Hess} f)=d \Delta f+\operatorname{Rc}(\nabla f, \cdot)=-d\left(R-c|d \phi|^{2}\right)+\operatorname{Rc}(\nabla f, \cdot) .
$$

Plugging into (2.3), we get

$$
\frac{1}{2} d R-c\left(\tau(\phi) d \phi+\frac{1}{2} d|d \phi|^{2}\right)-d\left(R-c|d \phi|^{2}\right)+\operatorname{Rc}(\nabla f, \cdot)=0,
$$

or

$$
-\frac{1}{2} d R+\frac{c}{2} d|d \phi|^{2}-c \tau(\phi) d \phi+\operatorname{Rc}(\nabla f, \cdot)=0 .
$$

Using $\tau(\phi)=d \phi(\nabla f)$, we finally have

$$
(\mathrm{Rc}-c d \phi \otimes d \phi)(\nabla f, \cdot)=\frac{1}{2} d\left(R-c|d \phi|^{2}\right) .
$$

According to our notations, this completes the proof.

The main result of this section is the following new identity.

Theorem 2.2 On a gradient $\mathrm{RH}$ soliton, there holds

$$
\Delta S+2|\mathscr{S}|^{2}-d S(\nabla f)+2 \sigma S+2 c|d \phi(\nabla f)|^{2}=0 .
$$


Proof By (1.2), one gets

$$
\operatorname{Rc}(\nabla f, \cdot)=c d \phi \otimes d \phi(\nabla f, \cdot)-\operatorname{Hess} f(\nabla f, \cdot)-\sigma d f .
$$

The above combined with (2.1) yields

$$
c d \phi \otimes d \phi(\nabla f, \cdot)-\operatorname{Hess} f(\nabla f, \cdot)-\sigma d f=\frac{1}{2} d S+c d \phi \otimes d \phi(\nabla f, \cdot) .
$$

Using $d|\nabla f|^{2}=2 \operatorname{Hess} f(\nabla f, \cdot)$, one gets

$$
d\left(S+|\nabla f|^{2}+2 \sigma f\right)=0 .
$$

Taking divergence of (2.6), we have

$$
\begin{aligned}
0 & =\Delta S+2 \sigma \Delta f+\Delta|\nabla f|^{2} \\
& =\Delta S+2 \sigma \Delta f+2|\operatorname{Hess} f|^{2}+2 \operatorname{Rc}(\nabla f, \nabla f)+2 d(\Delta f)(\nabla f) \\
& =\Delta S+2 \sigma(-S-m \sigma)+2|\mathcal{S}+\sigma g|^{2}+d S(\nabla f)+2 c d \phi \otimes d \phi(\nabla f, \nabla f)-2 d S(\nabla f) \\
& =\Delta S+2 \sigma S+2|\mathscr{S}|^{2}-d S(\nabla f)+2 c|d \phi(\nabla f)|^{2},
\end{aligned}
$$

where we used (2.1), or equivalently,

$$
\operatorname{Rc}(\nabla f, \cdot)=c d \phi \otimes d \phi(\nabla f, \cdot)+d S / 2
$$

for the third equality.

\section{On Compact Gradient RH Solitons}

In this section, we use (2.5) to prove results for compact solitons.

Theorem 3.1 Assume that $((M, g),(N, h), \phi, f, \sigma)$ is a gradient $\mathrm{RH}$ soliton. If $M$ is compact, then we have $S_{\min }=0$ in the steady case; in the shrinking case $0 \leq S_{\min } \leq-m \sigma$ and in particular $S \geq 0$; in the expanding case $-m \sigma \leq S_{\min } \leq 0$ and in particular $S \geq-m \sigma$.

Proof Using $|\mathscr{S}|^{2} \geq S^{2} / m$ and dropping the last term in (2.5), we get

$$
\Delta S+\frac{2}{m} S^{2}+2 \sigma S-d S(\nabla f) \leq 0 .
$$

When $M$ is compact, there is a point $p_{0} \in M$ such that $S$ attains its minimum $S_{\min }$ in $p_{0}$. At the point $p_{0}$, we have $\Delta S\left(p_{0}\right) \geq 0$ and $d S\left(p_{0}\right)=0$. By (3.1), we obtain

$$
\frac{2}{m} S_{\min }^{2}+2 \sigma S_{\min } \leq 0 \text {. }
$$

Thus if $\sigma=0$, we have $S_{\min }=0$. If $\sigma<0$, we have $0 \leq S_{\min } \leq-m \sigma$. If $\sigma>0$, we have $-m \sigma \leq S_{\min } \leq 0$.

Corollary 3.2 Assume that $M$ is compact and $((M, g),(N, h), \phi, f, \sigma)$ is a steady or expanding soliton, then $f$ is constant, $\phi$ is a harmonic map and $\mathrm{Rc}=c d \phi \otimes d \phi-\sigma g$.

Proof On gradient RH solitons, we have $\Delta f=-S-m \sigma$. In the steady case, one has $S \geq 0$ and $\sigma=0$ and thus $\Delta f \leq 0$. In the expanding case, one has $S \geq-m \sigma$ and thus $\Delta f=-S-m \sigma \leq 0$. Hence on either steady or expanding solitons, we find that $f$ is subharmonic. Since $M$ is compact, we get that $f$ must be a constant function. By $(1.3)$, then $\tau(\phi)=0$.

Remark 3.3 If $((M, g),(N, h), \phi, f, \sigma)$ is a steady $\mathrm{RH}$ soliton and $M$ is compact, then the Ricci curvature of $(M, g)$ must be strictly positive unless $\phi$ is a constant map. 
Unlike the steady or expanding case, for compact shrinking soliton the potential function is not necessarily a constant. However, we have the following observation.

Corollary 3.4 Assume that $M$ is compact and $((M, g),(N, h), \phi, f, \sigma)$ a shrinking soliton. If in addition $S_{\min }=-m \sigma$, then $f$ is constant, $\phi$ is harmonic, $\mathrm{Rc}=c d \phi \otimes d \phi-\sigma g$ and $S \equiv S_{\min }$. Proof If $S_{\min }=-m \sigma$, then $S \geq-m \sigma$ and thus $\Delta f=-S-m \sigma \leq 0$. Hence $f$ is constant. Plugging into the soliton equations, we get all conclusions.

\section{A Discussion on Generalization of Quasi-Einstein Manifolds}

In [3], Case-Shu-Wei introduced quasi-Einstein manifolds. A triple $\left(M^{m}, g, f\right)$ is called quasiEinstein if it satisfies the equation

$$
\mathrm{Rc}+\operatorname{Hess} f-\frac{1}{m} d f \otimes d f+\sigma g=0 .
$$

We notice that in the definition of RH soliton, if we drop condition (1.3) and consider only (1.2) itself, then this is a generalization of quasi-Einstein (4.1). For convenience, we call $\left(\left(M^{m}, g\right)\right.$, $\left.\left(N^{n}, h\right), \phi, f\right)$ a generalized quasi-Einstein manifold if it satisfies (1.2), i.e.,

$$
\operatorname{Rc}+\operatorname{Hess} f-c \phi^{*} h+\sigma g=0 .
$$

Here we do not require $c$ to be nonnegative.

We first consider a special case of (4.2) when $f$ is a constant. Note that the condition

$$
\operatorname{Rc}(g)=c \phi^{*} h-\sigma g
$$

is not a special case of the $\mathrm{RH}$ soliton by taking the potential function $f$ to be constant, since the $\mathrm{RH}$ soliton equation is a coupled equation system and the second equation will immediately imply $\phi$ is harmonic when $f$ is constant. Here we drop the second equation of the RH soliton, however we still have the following.

Lemma 4.1 Assume that $\phi:(M, g) \rightarrow(N, h)$ satisfies $\operatorname{Rc}(g)=c \phi^{*} h-\sigma g$ for $c \neq 0$. Then $\tau(\phi) d \phi=0$ as a one form, where $\tau(\phi) d \phi=h(\tau(\phi), d \phi \cdot)=h_{\alpha \beta} \tau^{\alpha}(\phi) \partial_{i} \phi^{\beta} d x^{i}$. Furthermore, if there is a vector field $X \in T(M)$ such that $\tau(\phi)=d \phi(X)$, then $\phi$ is harmonic.

Proof By Rc $=c \phi^{*} h-\sigma g$, one has $R=c|d \phi|^{2}-\sigma m$ and

$$
\frac{1}{2} d R=\operatorname{DivRc}=c \operatorname{Div} \phi^{*} h=c\left(\tau(\phi) d \phi+\frac{1}{2} d|d \phi|^{2}\right)=c \tau(\phi) d \phi+\frac{1}{2} d R .
$$

If $c \neq 0$, one gets $\tau(\phi) d \phi=0$. In addition, if $\tau(\phi)$ can be represented as $d \phi(X)$ for some $X$, then

$$
0=(\tau(\phi) d \phi)(X)=\tau(\phi)(d \phi(X))=|\tau(\phi)|^{2} .
$$

Thus $\phi$ is harmonic.

We present an immediate application of Lemma 4.1.

Corollary 4.2 Assume that $(M, g)$ has positive Ricci curvature Rc. Then the identity map $\mathrm{Id}:(M, g) \rightarrow(M, \mathrm{Rc})$ is harmonic. Similarly, if $(M, g)$ has negative $\mathrm{Rc}$, then $\mathrm{Id}:(M, g) \rightarrow$ $(M,-\mathrm{Rc})$ is harmonic.

Proof First note that $\mathrm{Id}:(M, g) \rightarrow(M, \pm \mathrm{Rc})$ satisfies $\mathrm{Rc}= \pm \mathrm{Id}^{*}( \pm \mathrm{Rc})$. Thus the assumption in Lemma 4.1 holds with $c= \pm 1$ and $\sigma=0$. Hence we have $\tau(\phi) d(\mathrm{Id})=0$, in local coordinates 
this means

$$
\pm R_{i j} \tau^{i}(\mathrm{Id}) d x^{j}=0
$$

Definiteness of the Ricci tensor then implies $\tau^{i}(\mathrm{Id})=0$ for any $i$, hence Id is harmonic.

Recall that any Riemannian manifold $M$ of dimension $m \geq 3$ carries a complete metric with negative Ricci curvature, see [9]. Thus one cannot say anything about the manifold $M$ without restrictions on the target manifold $(N, h)$. In (4.3), the target manifold plays an essential rule.

\section{Appendix}

Assume that $(M, g)$ and $(N, h)$ are two Riemannian manifolds and $\phi: M \rightarrow N$ a map. In this appendix, we prove formula (2.4) using local coordinates, i.e.,

$$
\operatorname{Div}(d \phi \otimes d \phi)=\tau(\phi) d \phi+\frac{1}{2} d|d \phi|^{2} .
$$

Recall that in our notation $d \phi \otimes d \phi=\phi^{*} h$. For any $p \in M$, let $\left\{x^{i}\right\}_{i=1}^{m}$ be local coordinates orthonormal at $p$, and let $\left\{\phi^{\alpha}\right\}_{\alpha=1}^{n}$ be local coordinates orthonormal at $\phi(p) \in N$. We use $\Gamma_{i j}^{k}$ and $\Gamma_{\alpha \beta}^{\gamma}$ to denote Christoffel symbols of $M$ and $N$, respectively. Let $\partial_{i} \doteqdot \frac{\partial}{\partial x^{i}}$ and $\partial_{\alpha} \doteqdot \frac{\partial}{\partial \phi^{\alpha}}$. We use Einstein convention of implicit summation over repeated indices. By definition, at the point $p$ we get

$$
\tau^{\alpha}(\phi)=\partial_{i} \partial_{i} \phi^{\alpha}-\Gamma_{i i}^{j} \partial_{j} \phi^{\alpha}+\Gamma_{\beta \gamma}^{\alpha} \partial_{i} \phi^{\beta} \partial_{i} \phi^{\gamma}
$$

At $p$, we then obtain

$$
\begin{aligned}
\operatorname{Div}(d \phi \otimes d \phi)\left(\partial_{i}\right)= & \left(\nabla_{j}(d \phi \otimes d \phi)\right)\left(\partial_{j}, \partial_{i}\right) \\
= & \partial_{j}\left(d \phi \otimes d \phi\left(\partial_{j}, \partial_{i}\right)\right)-d \phi \otimes d \phi\left(\nabla_{j} \partial_{j}, \partial_{i}\right)-d \phi \otimes d \phi\left(\partial_{j}, \nabla_{j} \partial_{i}\right) \\
= & \partial_{j}\left(h_{\alpha \beta} \partial_{j} \phi^{\alpha} \partial_{i} \phi^{\beta}\right)-\Gamma_{j j}^{k} \partial_{k} \phi^{\alpha} \partial_{i} \phi^{\alpha}-\Gamma_{j i}^{k} \partial_{j} \phi^{\alpha} \partial_{k} \phi^{\alpha} \\
= & \partial_{j} \phi^{\gamma} \partial_{\gamma}\left(h_{\alpha \beta}\right) \partial_{j} \phi^{\alpha} \partial_{i} \phi^{\beta}+\partial_{j} \partial_{j} \phi^{\alpha} \partial_{i} \phi^{\alpha}+\partial_{j} \phi^{\alpha} \partial_{j} \partial_{i} \phi^{\alpha} \\
& -\Gamma_{j j}^{k} \partial_{k} \phi^{\alpha} \partial_{i} \phi^{\alpha}-\Gamma_{j i}^{k} \partial_{j} \phi^{\alpha} \partial_{k} \phi^{\alpha} \\
= & \left(\Gamma_{\gamma \alpha}^{\beta}+\Gamma_{\gamma \beta}^{\alpha}\right) \partial_{j} \phi^{\gamma} \partial_{j} \phi^{\alpha} \partial_{i} \phi^{\beta}+\partial_{j} \partial_{j} \phi^{\beta} \partial_{i} \phi^{\beta}+\partial_{j} \phi^{\alpha} \partial_{j} \partial_{i} \phi^{\alpha} \\
& -\Gamma_{j j}^{k} \partial_{k} \phi^{\beta} \partial_{i} \phi^{\beta}-\Gamma_{j i}^{k} \partial_{j} \phi^{\alpha} \partial_{k} \phi^{\alpha} \\
= & \left(\Gamma_{\gamma \alpha}^{\beta} \partial_{j} \phi^{\gamma} \partial_{j} \phi^{\alpha}+\partial_{j} \partial_{j} \phi^{\beta}-\Gamma_{j j}^{k} \partial_{k} \phi^{\beta}\right) \partial_{i} \phi^{\beta} \\
& +\Gamma_{\gamma \beta}^{\alpha} \partial_{j} \phi^{\gamma} \partial_{j} \phi^{\alpha} \partial_{i} \phi^{\beta}+\partial_{j} \phi^{\alpha} \partial_{j} \partial_{i} \phi^{\alpha}-\Gamma_{j i}^{k} \partial_{j} \phi^{\alpha} \partial_{k} \phi^{\alpha} .
\end{aligned}
$$

In the last equality, the first line is $\tau^{\beta}(\phi) \partial_{i} \phi^{\beta}$. For the second line, we calculate at the point $p$ :

$$
\begin{aligned}
\partial_{i}\|d \phi\|^{2} & =\partial_{i}\left(g^{k l} h_{\alpha \beta} \partial_{k} \phi^{\alpha} \partial_{l} \phi^{\beta}\right) \\
& =\partial_{i}\left(g^{k l}\right) \partial_{k} \phi^{\alpha} \partial_{l} \phi^{\alpha}+\partial_{\gamma}\left(h_{\alpha \beta}\right) \partial_{i} \phi^{\gamma} \partial_{k} \phi^{\alpha} \partial_{k} \phi^{\beta}+2 \partial_{i}\left(\partial_{k} \phi^{\alpha}\right) \partial_{k} \phi^{\alpha} \\
& =-2 \Gamma_{k i}^{l} \partial_{k} \phi^{\alpha} \partial_{l} \phi^{\alpha}+2 \Gamma_{\gamma \alpha}^{\beta} \partial_{i} \phi^{\gamma} \partial_{k} \phi^{\alpha} \partial_{k} \phi^{\beta}+2 \partial_{i}\left(\partial_{k} \phi^{\alpha}\right) \partial_{k} \phi^{\alpha} .
\end{aligned}
$$

Thus, we have

$$
\operatorname{Div}(d \phi \otimes d \phi)\left(\partial_{i}\right)=\tau^{\beta}(\phi) \partial_{i} \phi^{\beta}+\frac{1}{2} \partial_{i}|d \phi|^{2}=\left(\tau(\phi) d \phi+\frac{1}{2} d|d \phi|^{2}\right)\left(\partial_{i}\right) .
$$




\section{References}

[1] Aubin, T.: Some Nonlinear Problems in Riemannian Geometry, Springer Monographs in Mathematics, Springer-Verlag, Berlin, 1998

[2] Barros A., Ribeiro, Jr., E.: Some characterizations for compact almost Ricci solitons. Proc. Amer. Math. Soc., 140(3), 1033-1040 (2012)

[3] Case, J., Shu, Y.-J., Wei, G.: Rigidity of quasi-Einstein metrics. Differential Geom. Appl., 29(1), 93-100 (2011)

[4] Chen, B.-L., Gu, H.-L.: Canonical solitons associated with generalized Ricci flows. Sci. China Math., 56(10), 2007-2013 (2013)

[5] Chow, B., Lu, P., Ni, L.: Hamilton's Ricci flow, Graduate Studies in Mathematics, vol. 77, American Mathematical Society, Providence, RI; Science Press, New York, 2006

[6] Guo, H., Philipowski, R., Thalmaier, A.: A stochastic approach to the harmonic map heat flow on manifolds with time-dependent Riemannian metric. Stochastic Processes Appl., 124, 3535-3552 (2014)

[7] Jauregui, J. L., Wylie, W.: Conformal diffeomorphisms of gradient Ricci solitons and generalized quasiEinstein manifolds. J. Geom. Anal., 25(1), 668-708 (2015)

[8] Li, Y.: Eigenvalues and entropies under the harmonic-Ricci flow. Pacific J. Math., 267(1), 141-184 (2014)

[9] Lohkamp, J.: Metrics of negative Ricci curvature. Ann. of Math. (2), 140(3), 655-683 (1994)

[10] Müller, R.: Ricci flow coupled with harmonic map heat flow (PhD thesis), Eidgenössische Technische Hochschule ETH Zürich, DOI: 10.3929/ethz-a-005842361, 2009

[11] Müller, R.: Ricci flow coupled with harmonic map flow. Ann. Sci. Éc. Norm. Supér. (4), 45(1), 101-142 (2012)

[12] Pigola, S., Rigoli, M., Rimoldi, M., et al.: Ricci almost solitons. Ann. Sc. Norm. Super. Pisa Cl. Sci. (5), 10(4), 757-799 (2011)

[13] Williams, M. B.: Results on coupled Ricci and harmonic map flows. Adv. Geom., 15(1), 7-26 (2015)

[14] Yang, F., Shen, J.-F.: Volume growth for gradient shrinking solitons of Ricci-harmonic flow. Sci. China Math., 55(6), 1221-1228 (2012) 